\title{
SU ALCLNI ASPETTI CARATTERISTICI DEI MICROSISMI \\ A ROMA IN RELAZIONE CON FATTORI METEOROLOGICI
}

\author{
Milnizio Gimisi
}

Sotto il nome generico di mi(ro-i-mi vanno rompresi lumi i mo. vimenti del suolo rilevabili con $i$ sismografi e dovuti alle ratser pii diverse, e che d'altra parte hamno un aspetto raralteristico ben di stinguibile, a seconda della loro origine. Particolare interesse hammo quei microsismi che costituiseno un'igitazione del solo a rarallere permanente, più o meno regolare, di periodo e di ampiezza variabili entro ampi limiti. Essi sono in stetta relszione con patrolari fattori meteorologici, agenti anche molto lontano dal luogo di oserviatone: h.mmo una durata che va da qualelere ora fino a diveri giorni. eonstano di onde elastiche progresive di tipo superficiale propaganti-i con velocita di $2.5-4 \mathrm{~km} /-\mathrm{ec}$, dipendente dalla natura del mezzo at traversato. Il notevole effetto di di-persone rhe si rerifica nel rampe dei periodi (1 see-10 sec) propri delle onde mirro-ismiehe e il diverso assorbimento dei vari periodi sono le principali cau-e che inpediscono l̈identifucazione dei singoli treni donda in O-nervatori distanti tra loro più di qualehe chilometro.

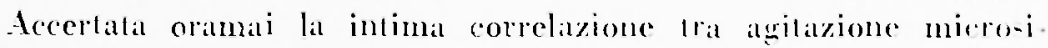
smica e perturbazioni atmoseriche $(1,-)$ is necessalio perfezionate la leenica del loro impiego per la individuazione dei centri delle pere turbazioni stesee, per determinare il dinami-no di que-li e soprattutto per prevederne i movimenti e levoluzione. I miero-i-mi. infatli, offrono lit posibilita di fare owervazioni rontinue di gromo e di notte e consentono una previsione, in alemi casi, notevolmente anticipata rispetto ai metodi piu moderni della meleorologial le indagini escruile con il loro am-ilio po-sono spingersi fino a zone distanti qualehe migliaio di thilometri dal luogo di o-ecriazione, mentre per esempio, le esplorazioni dei cicloni e denti uragame col radar hammo un raggrio effuale minore di 200 ("). Yaturalmente non è da pensare che i rilevanenti mirro-i-midei pu- 
-ano inlegralmente sostinure i mezzi di indagine e di sludio fino ad ora adoperali nei problemi dellat meleorologia dinamira mal, senza dubbio, possono rostiture un aluto di printaria importanza; lat valubzione dellallivià dei rentri di perturbazioni atmo-feriehe falla mediante rileramenti mierosismici assume, infalli, un signifieato ed una portala ben diversa dille elasiche misure delle grandezze rhe inleresinno lat meleorologia. Quese per lo piò si riferiscono solo al luogo di onervazione c soltanto con un gram numero di osscrvazioni -ingoble a di-continue nel tempo e nello spazio elfelluate rontemporaneamente os posibile dedure, previa una areurala elabolazione eseEnila da persomale specializzalo, ma deteminazione dello stalo del lempo e dellat -lla probabile evoluzione in una rertat regione; i rile-

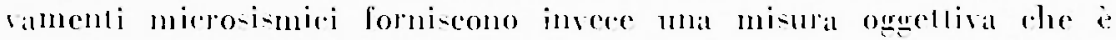
il ri-ultalo globale dell'allivila dei centri di perturbazione in una regione, anche molo extesil, allomo al luogo di oservazione r rhe dal sola puib ro-lituire un elemento indiration di grande officarial.

Vell-Juliuto Nazionale di Geofisicat, mediante il maleriale di os.

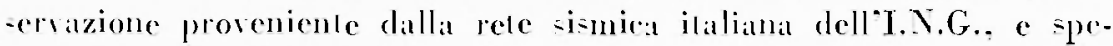
rialmente ron lan-ilio dell'Os-cervalorio Centrale di Romal, si slal condurendo uno -tudio sislemalico dei mierosismi in relazione ron le

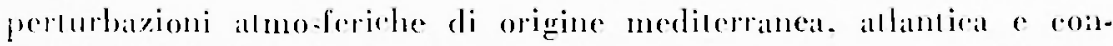
linchlale. al fine di ragegiungere mat mignliote ronosecnza dellat interdipendenzal di quaceli fenomeni.

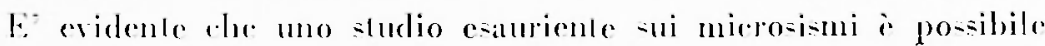
-olo se -i hamno a disposizione simografi adalli a forle ingrandimen-

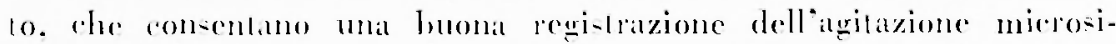

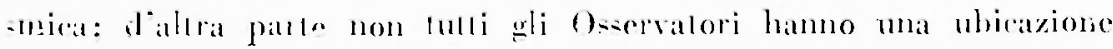
epportuna per que-lo genere di ricerches vi sono localitit rhe risultano parlicolamente farorevoli a reue-10 seopo.

I miero-ismi dinendono, infalti, in manielat cosprenat, dallo spe-s -ore e dalla nalura delle formazioni ereologrehe piò e-lene. purehs di

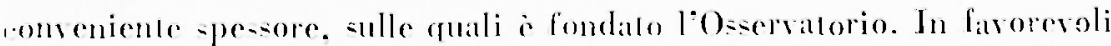

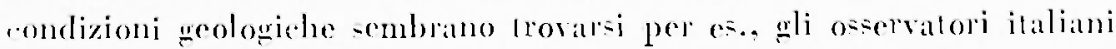
di Romal. Padoral e Calanial michosimi molto stiluppati in questi

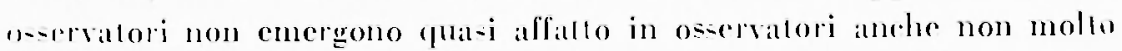
lonlani da esi, come, per es, arviene per Mesinal e Salò prosinue ri-pellivamenle a Calania o Palova. Naluralmente qui si intende par. 


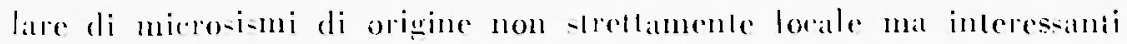

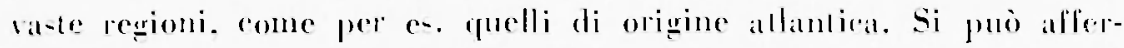

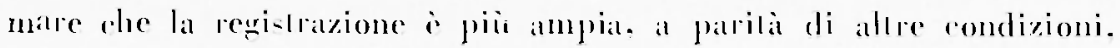
negli osecralori siluali -n formazioni greologiche piò resenti. e spe-

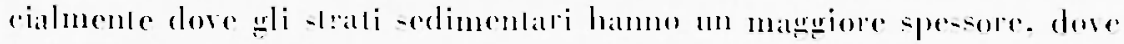

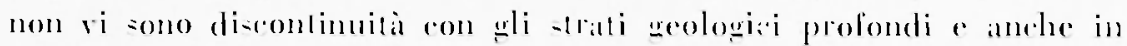

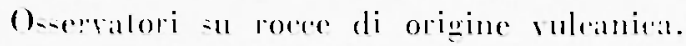

K. Sezawil ('). T'. Suzuki ('). A. II. Lee ("). nel ra-o di onde -mperficiali del tipe di Rayleigh. hamno sudialo ledlello di -lratifi-

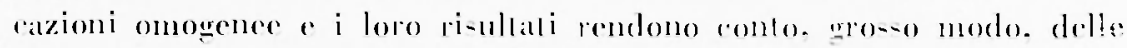
anomalis ele se risontrano nei riguade delle caralleri-liche di que-

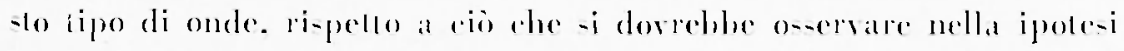

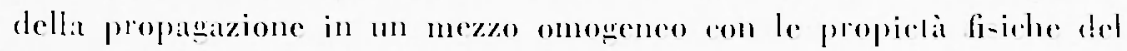

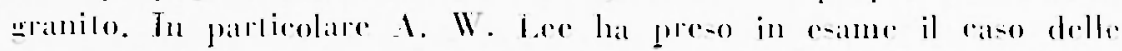
onde di Rayleigh in un mezzo arente al di -opra nno -lralo -uperfireiale comprimibile fprecisanente granilo ron -lrato sorrappo-to di

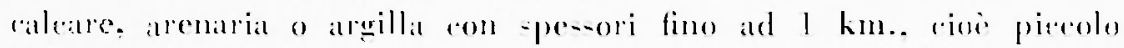

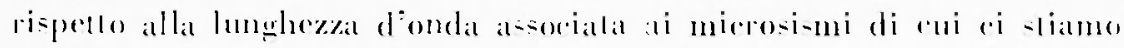
oreupandos: il rapporto dellampiezza orizzontale ri-pello a fuella verlicate non is rostanle, eome per le onde di Reg̣leight in un mezzo omogeneo. ma i fumzione della lumghezzat donda e dello -penene

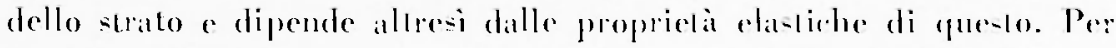
- Mati sollili que-lo rapporlo pmò rereere da 0.68 a 1.35, (le am-

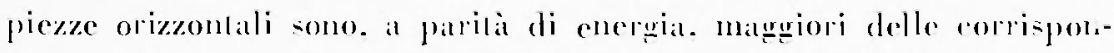
denli ampiezze delle onde di Raỵleigh nel aranilot. l’influenza dello sralo ò magrione -ni movimenli orizzonlali che -ni movimenli veliciali.

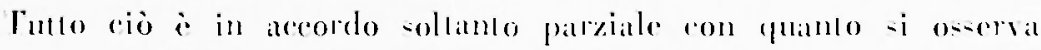
in pratiral, e limitatamente al raso di movimenti mirrosimbei arenti

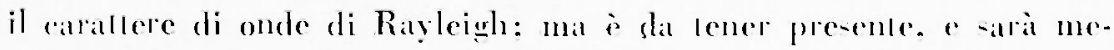
grio precisalto in mat prosima romuniraxione, che i micro-i-ni non consiano sempre o sollanto di onde di Raygleigh perehe a rolle presentano invere tmo precalo ratrallere di vibraxione langenziale. prove prio delle onde di love. Un decennio di oservazioni preso l'o-serva. forio centrale dell'Jsiluto Kazionale di Geofisica con-ente di alfermare. e la questione verra approfondila nel proseguimento di questo -ludio sui microsismi, rhe la nama delle onde clastirhe assoriate ai mirrosismi caria a seconda della ransa rhe da ad essi origine, perché 
diverso i forse il meccanismo con il yuale esse ingono provocate ne! suolo (microsioni dovuti al vento, allaxione dei marosi contro le roste, all'allivilà dei reicloni, a fronti freddi, cere).

I risulatli delle owervizioni conlermano pure rhe enli Okervallori in poizione geologieanente favorevole per la regi-trazione dei mierosi-mi consentono una huona regi-lrazione per lutli i lipi di mierosi-mi.

Da un ronfronto e-entuito -ul malleriale di o-crvazione di rirat ollo mesi (febbrato-ollobre 19-48) di aleme O-servaltori della rete -ismica dell't-liluto Xaxionale di Geofisical (Padova, Salo. Firenze. Bo-

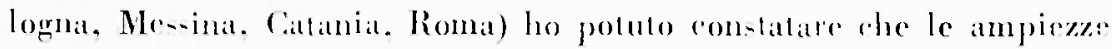

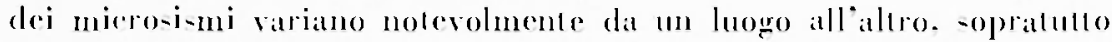
a catll-al della natura o dellat trultura leltonica dell'immediato sottosuolo sll rui poggia l'oservalorio, oltre rhe della relativa distanza del rentro di perlurbazione. Dathra parte it decadimento dellenergia nella propagazione i lento: infitli tratlandosi di onde di tipo-tuperfieiale, le ampiezze dererecono eponenzialmente con la profondita

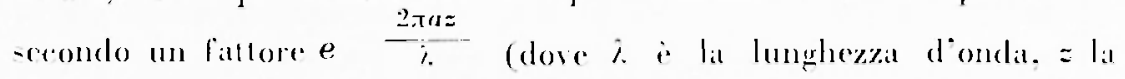
profondita e a $\dot{e}$ un fittore, dipendente di mezzo. al quale Gutenler dit il valore 0.3) a quindi la propagazione aviene in uno slato dello -pesore di rirea una lumphezza dondat. (una rentina di km rirea pere i periodi più wrandi). Forti arosthmenti o brusehi deradimenti dellenergia a-sosiala ai miero-i-mi vamo dumque allribuiti a cherogeneita della rrosta lungo il tragitto di propagazione e a dienontinuil lra blocedie peologici diver-i.

l: - tato eneruito un ronfronto fra alcuni degli onecratori sopra detli. prendendo per riasemo un valore medio dell ampiezza owere

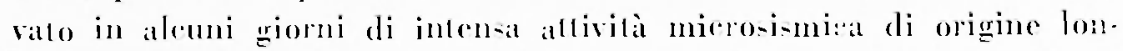
lana nel periodo di tempo sopra ricordato. Falla tughale ad l l'ampitza dei mirero-i-mi che si o-ervano a Romil. -i hamno per le altre starioni i -renuenti valori:

\begin{tabular}{|c|c|c|c|c|}
\hline Rolul & C $\triangle T \backslash \backslash 11$ & PАINМ & SALOH & MESSIXI \\
\hline 1. & 1 & $(0,8$ & 0.5 & $0 .-4$ \\
\hline
\end{tabular}

Una variazione del periodo è risultata molto meno evidente.

Il salore eceezionalmente pircolo rhe si hat per Mesina potrebbe allubuir-i a probabili discontinuila geologrehe ron gli strati piò profondi ed allat mincanza di stati alluvionali sufferientemente eslesi c profondi. 
Loservalorio di Roma, Pondalo su rerreno di riporlo somra. tamle al lubo litoide ("), i particotarmente adallo per lo studio dei microsini in genere. o in particolare di fuelli in relazione con lo

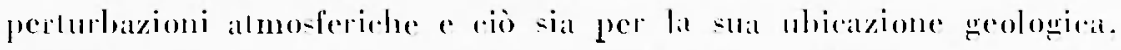
notevolmente farorevole a questo riguardo, sia per la sua oltinat doe lazione strumentale foperialmente ulili risultano per lo sudio dei microsioni tre strumenti Galizin-Wilip a renistazione folograferes. due per le compronemli orizzontali ed uno per la verlieale funzionami con un periodo di circa $10^{*}$, e due strumenti orizzontali elellromaEnclici 1.N.G. lipo Galizin con un periodo di rirra 2.19) -ia ancora

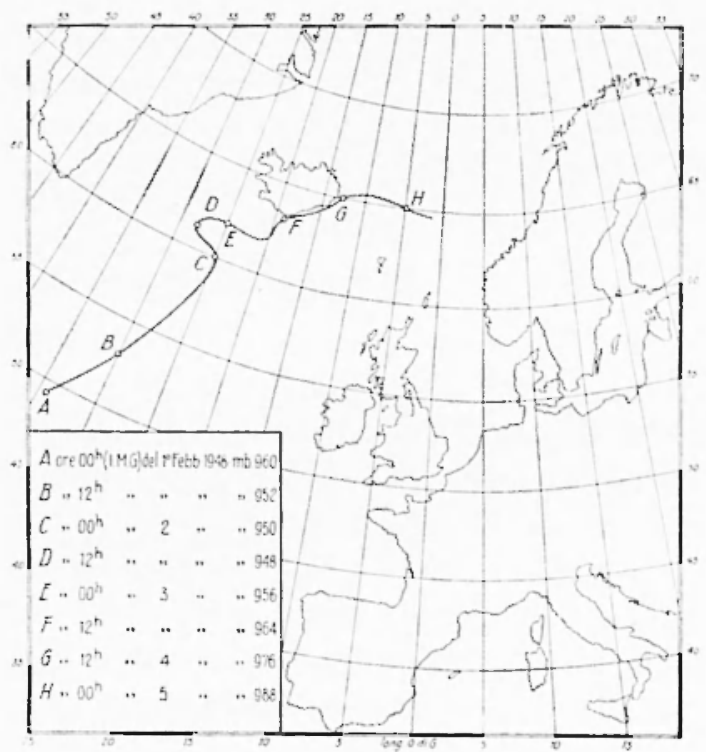

Fig. 1 sopratlullo. prer las slla partiroliter posizions. weogrativa in pieno hatino medilerrineo.

Per quanto latgilazione micro-i-miea pre -coli a volte in que-to Deserallorio mal strullurat romplinati-imat per la sorrapponizione di microsismi di annpiezze e periodi difl.renti provenienti di

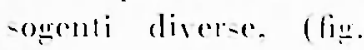
9). -opratullto a call-al dellar romplenila dellit rewione dal punto di vista meleorologicos, $i$ lerilo lullarial. in hat-1

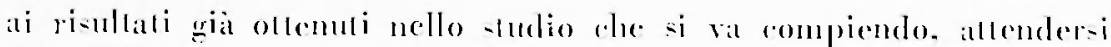
dai dati microsismiei un effeate eontributo al problema della previsione del tempo, sperialmente per le regioni allorno al hateino mediterraneo.

Falla astrazione dall'ampiezat, dalla quale si può dechure inna valuazione dellentensila mierosimiea in relazione eon lat allivila. la whicazione e la esensione della sorgente da rui l'augitazione miero-i-

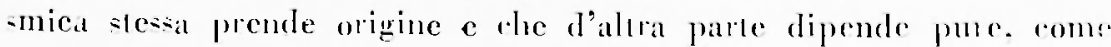
si è delto, dalla natura geologica degli strati solto-lanti al luogro di

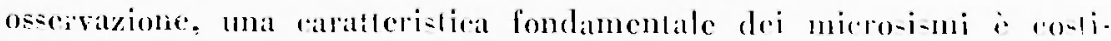




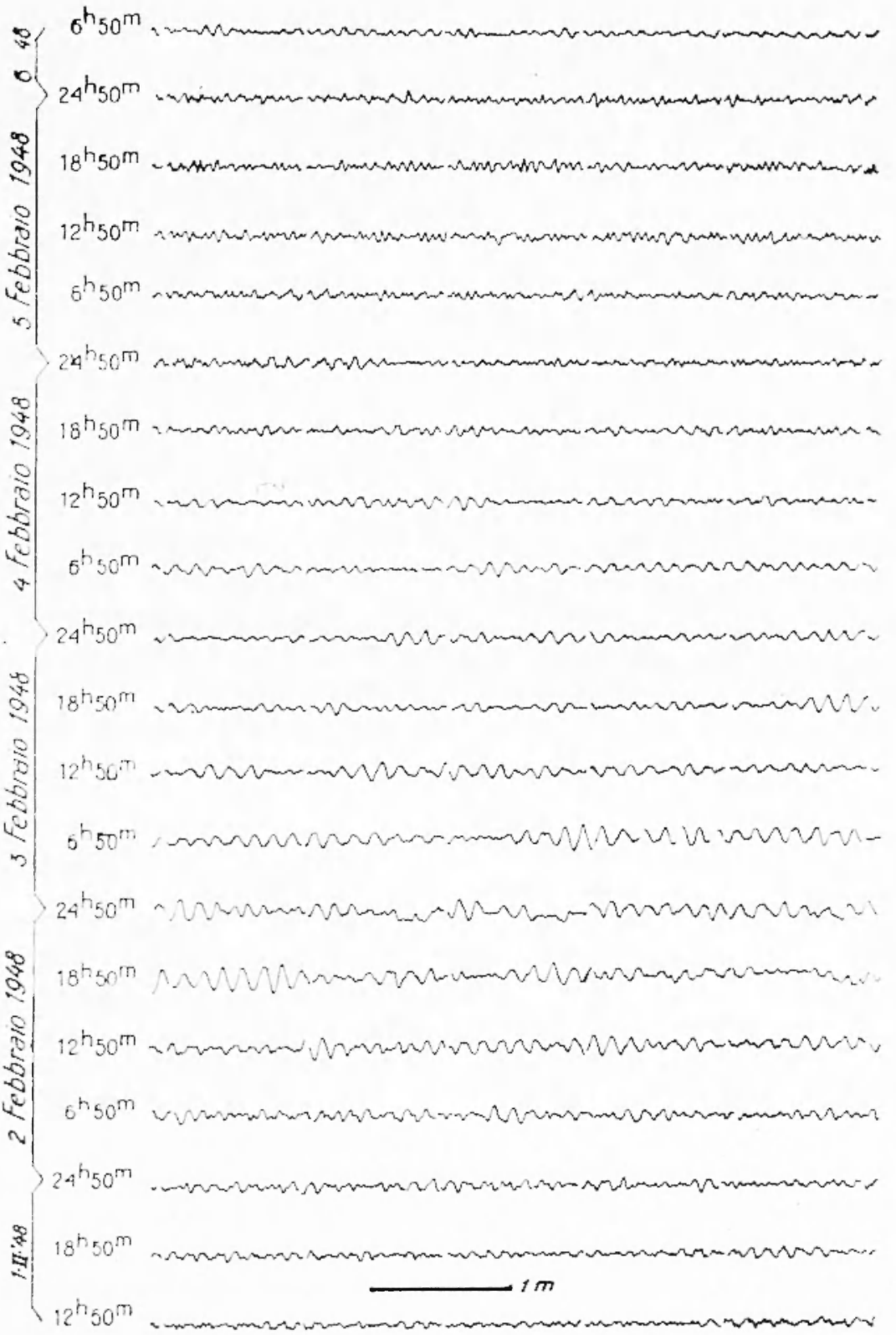

Fig. 2 
tuila dal periodo con cui esi si manilestino e che $\dot{a}$ in stretlat relazione con le ratuse dei mierosismi stessi e con il luog̣o donde provengono. E distingubilisima ma agitazione mierominnica di tipo medilerraneo da unaltra, per es. di origine atlantica fredi ligror. 2 , $8.11)$ ed $\dot{a}$ preciomente la grande differenzal dei periodi che consente: una prima interpretazione immediatas per la prima infatti il periodo

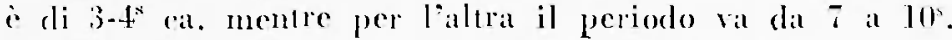

Mirro-ismi con periodi di $1^{4}, 5$ fino a 23,5 risultano di origine locale e si risontrano in concomilanza di fordi venti e mare grosso nel Tir-

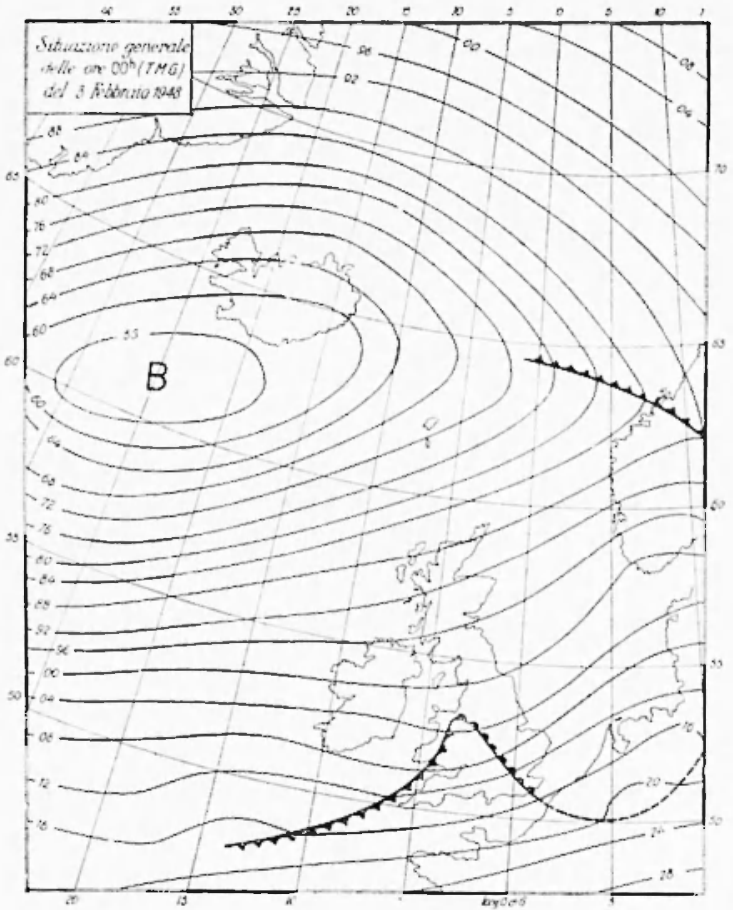

Fig. 3 reno rentrale fino al largo dellat Sirdegrna; il periodo aumenta man mano rle l'origrine -i allontanil.

Microsismi ('o n periodi di $l^{\text {s }}$ fino a $2 * . ;$ dellit durita di poche ore si verificano in concomilinzil di for i perturbazioni temporaleache locali a nell'arilazione microsismica prevale sen-ibilmente la romponente verticale. Anche qui il periodo ammenla matn mano rlae la presuntal origine si allonlina.

Mirrosimi r. on periodi di $3^{*}$ (a) -i hammo in corri-pondenza di forti perturbazioni -ul mare al largo del golfo di Genova in direzione sud-ovest, rioè nella zona di mare più profonda.

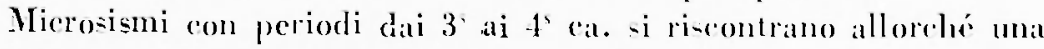
depresione sovrata il Tirreno centro-meridionale o ancle quando un fronte freddo attlaversal $l_{a}$ slessal regione regione. (Vedi figg. 2, 8).

Microsimi con periodi fino a $5^{\text {s }}$ ca. - i hamno quando la zona di perturhazione atmo-fericia inve-te il mediterranco orcidentale ed m ecntro ciclonico di ma certa altivila sovrata il male fra le Balcari 
c la Sardegna, oppune quando profonde perlurbazioni anmo-feriche -i hanno nello Ionio e nel Medilerraneo rentrale.

Quando una profondal depresione sovratal il mare aperto neil'Aliantico centro-scllentrionale si regislano a Romal microsismi più o memo ampi, sccondo l’inlensita della depresione tesal, (redi fiğ. 2) ron periodi che vanno da 7"al. funo ad un ma-imo di 9"-30"; il periodo aumenta man mano che la depresione va spostando-i dal centro delI'Allantico verso il nord in vicinanza delle co-le meridionali della Groenlandia (fino a 8-9" (a.); lornet poi lievemente a diminuire quan. do il cielone si inoltra crentualmente nello -trelto di Danimalrea tra l'TAtandia e le cose orientali della Groenlandia. Si nota pure che l'agitazione diminuice noterolmenle man mano whe la depresione almoferior invale la lerra ferma; il periodo diminuise aneor più

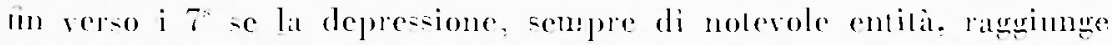
il matr di Norregia: diviene anche di 6 , mal lampiezzal in genere

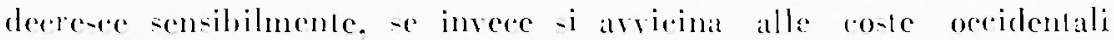
della Scoxia e della fran Brelligna. Si può dipe insomma rhe il perioflo hat un andimento revente con la dielanzal presindendo dalla probabile influenza rhe powouso avere sia lat calloal che dà ori-

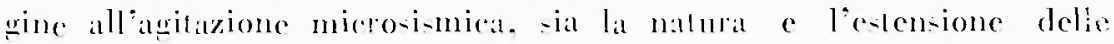
zone ceritate, questo fatto puis spexarsi tenendo presente il magqiore assorbimento rhe -ubiceono i presodi piu piecoli nelle onde di lipo

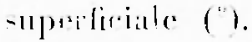

Riferendori sempre ai mirro-i-mi asociali a parlicolari permehazioni meicorolowiche si nota rhe le onde hammo min ratrallere piuttomo regolater fuando provengono da un mnical orgente (x. figrg. 2-11). Naluralnente di solilo si hammo siluarioni meleorologithe molso compliate rhe dimno luogo a sovrappo-izioni molepliat di microsiomi sempre della sessal nalutar mal con proveninze diverse (redi fig. 9): ne risulta quindi mat axiazione microsismiat intrirala in cul non is semplice la de. laminazione dei periodi componenti. Soltanto ron ma lunga epericnza si riene al deleminalre

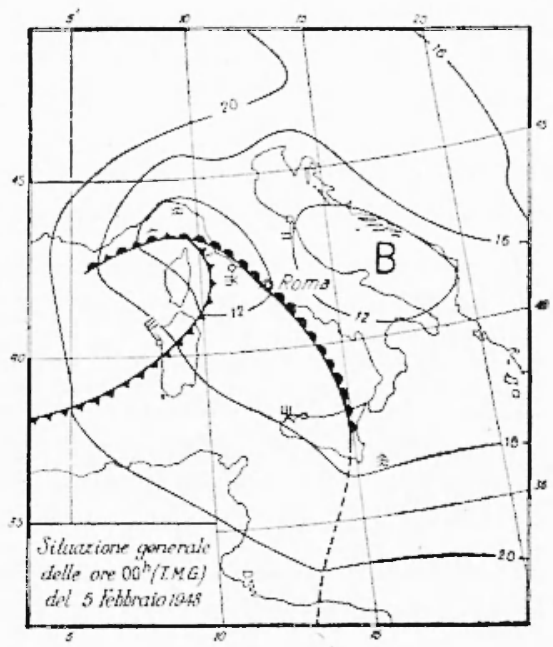

Fig. 4 
le fari componenti e a districate siluazioni ande mollo romplesse. Tha medesima siluazione meteorologiea, d'altra parle, comi-ponde in

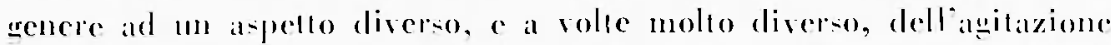
mierosimicat nei vari acervalori. Se si vonliono dumpe clementi si-

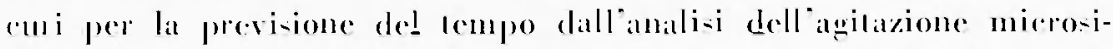
smica, oceore studiare le singole siluazioni meteorologishe in corri-pondenza dei miero-ismi, Osoervalorio per Osecrattorio perehie, eome

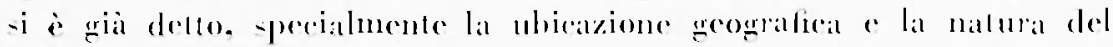

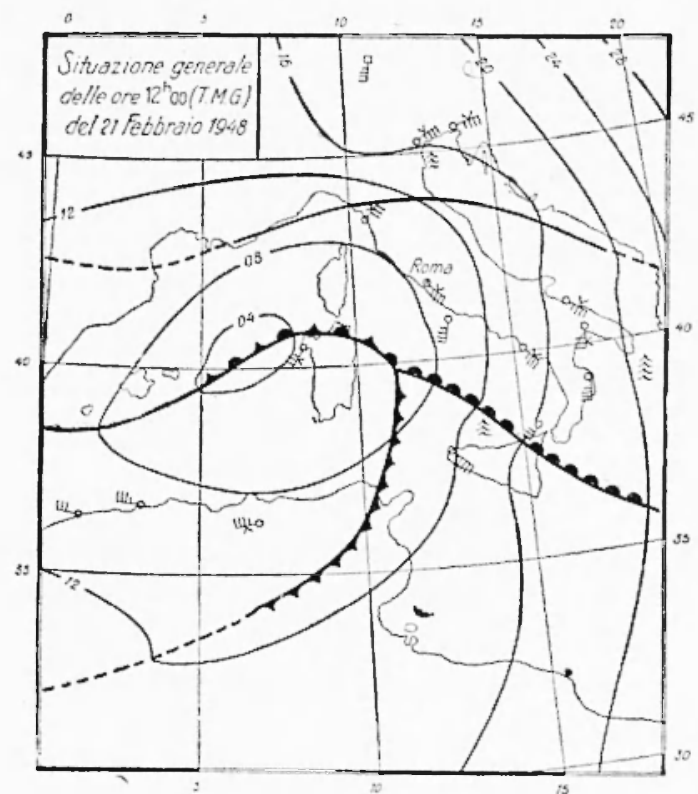

Fig. 5 sollosmolo supertisiale protondo alterano note. volmenle l'alspello delliagitazione micro-i-milea - lessat. Nello slesol lempo, oceorre seguire dellatElialimente la rompletia rooluzione delle perlurhazioni. dalla loro formalzione alla loroestinzione. cenendo presenti lulti po-sibili dali meoli-ieiper inalividuare en areertare le probabili rallos restra lerentri di lutli quenli lenomeni.

fi' necesiario peral. tro fare nono-ludio resro-pellivo. pere tentare di seoprire il legame lra mi-

crosismi e fallori greofisici di altra natura. onde sabilime le disere genze e lo correlazioni più o nono evidenti las i primi ed i secondi c determinare le diflerenziazioni caratleri-tiche delle diverse hurrasche mierosismiche, atte a rintraceiate le vere eaune ehe le producono.

Riportiamo ora i risultati dell'esame di qualehe lempe-sa mierosismica. I dali meteorologici relativi, per cortese rones-ione, sono stati prelevali dagli arehivi del Servizio Metcorologieo dell Acronautira italiana.

1) Tempesta microsismica del 31 gemaio-6 febbraio 19.18; cosii. tuise un eempio di agitazione mierosismica di origine atlantieas a 
cui si sovrappone poi un altra di origine mediteranea. La fiğ. ]. di le suecessive fasi dellevoluzione del econtro depresionario allantico in corri-pondenza de!la tempesa mierosismica.

Il I" fobbraio 19.18 un rentro depresionario, ele grià in precedenza -i cra delineato sull' Alantico entro-settentrionale, vat approfondendosi, si mosra pallicolarmente altivo, ed investe tulto l'Allantico settentronale. Si hamo renli forti e fortisimi $\left(8^{\prime \prime}\right.$ e $9^{\prime \prime}$ grado della -ala Beaufort) in lulla quesa zona e su lutte le coste atlantiobe dell'Europa. in Istanda $c$ a sud della Groenalandia. Il mal tempo imper. versa su lulto l'Alantico nord orientale. Vella figr. 2 sono riportati. a inlervalli di 6 ore, 5 minuli di rengistrazione mierosi-mica

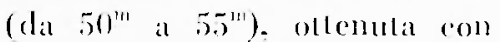
il sismorrafo eleltromagnetieo Galitzin-Wilip oricmato in direxione nord-sud. (l "intervallo i. -Lato scello per comodita di riproduzione fologralicar. dila la favorerole posizione ocempala nei -i-mogrammi dalla serie di lutli wli inlervalli). In esa -i puir redere reles - 11 un algitatzione di minor periodo di origine limenicas, va comparendo semper piò dislintal lagritazione di $7-8$ scoondi in relazione alla suddellat perturbazione at-

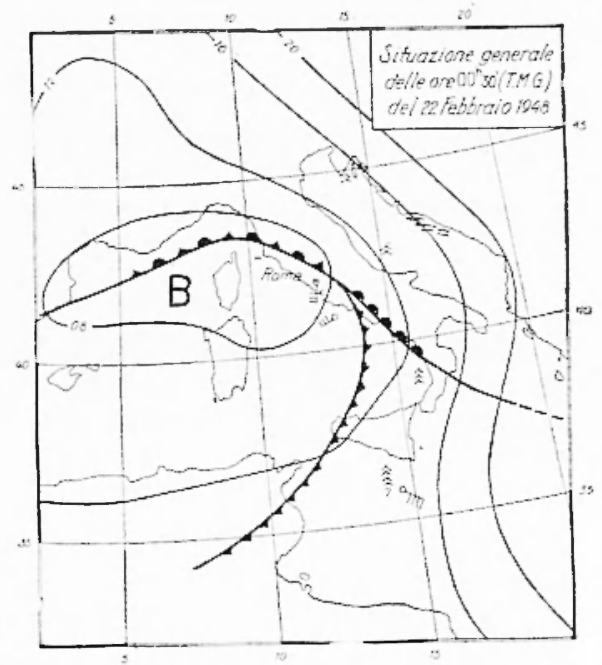

Fig. 6 lantira. La fig. 3 da la siluazione apencrale alle ore $60^{\mathrm{h}}$ (T.M. Th.) del 3. II. 48. In questo periodo di tempro la perturbazione rageriumese il massimo dinamiomo e nella fig. I s notano in guesto slemo lempo le massime ampiezze, i massimi prerodi (9.a.) o tun andamento più regolare. Il giorno 3 la deperesione aceoma a colmarsi gradualmente e seguita a spotarsi rerso l'Fslanda meridionile. Il griorno 4 si aceentual questa tendenza " proserue lo spostamento in direzione est: corrisponslenlemente l'agrilazione mierosismies decrese di intensiti, specialmente quando la depressione comineia in parte a insestire la terma ferma. Il giomo 5 il centro depressionario si e notevolmente ridolto ed ha ragrgimto in picno il mar di Norregia: l"ampiezzal decresce meno di quanto di s potrebbe allendere per la diminuila effeienza dinamiea del centeo 
=leso. Que-to minore desaldimente dell energial mierosi-miea ri-pelle

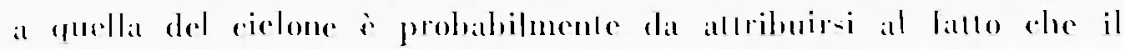
centro deprentonatio, se hat in effelli un minor dintmismo, is peri

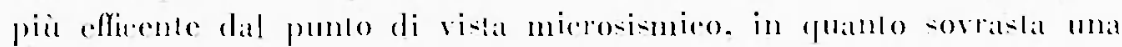

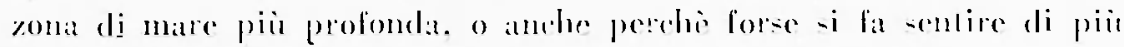
l'effello dei maro-i eontro le ro-le della Norreqia.

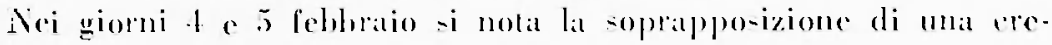

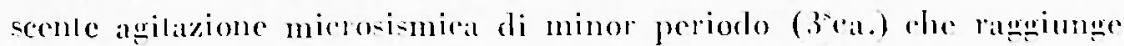

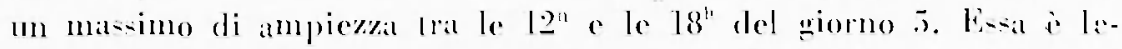
gatla allatlivili di un fronte freddo arenle del resto strelli lewani

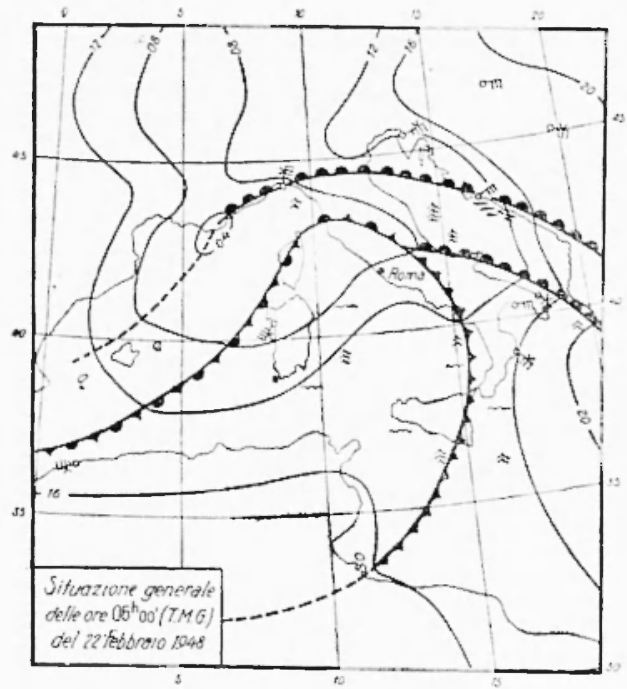

iig. 7 ron la perturbatione norelallanlica di rui -opral alello fronte enenclente-i prima in un lumgro arao dai l'iremai. allratrerso le rowe medilierrance francesi. fino alle Alpi Oecidentali, ha poi allratrer-alo in direzione-malsurlest il Merliterraneo ed il Timeno. la mageror atlisila micro-ismilal si osectat fluando il fromle is -11 ! Timeno entro-merialionale. lat fige 1 dil lat siluaxione erenerale sul Medilerranes alle $(10)^{\text {h }}$ (T.Ml. loricio 19.18.

2) Trmpesta microsismica arl 27.24 febbrain 19.18. I: una inle. resentle leslimonianza di agilazione di lipo medilerraneo. Alle on del giorno 2t. febhraio un rentro depresionario di non exesesira pro-

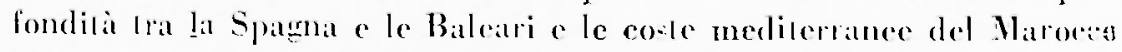
francese, va spostandosi in direzione est: la perturbazione meleoro-

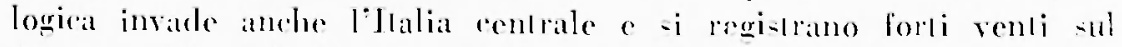
Tirreno. Alle 13" il ecnlro di basa pressione si e spostallo ald oves della Sardegna es ha un gradiente bariog piulloslo forle sul Tirreno

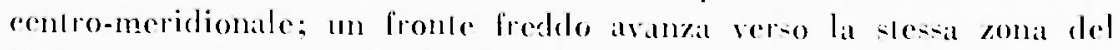
Tirrens, -i hammo renti for:isimi a raffiche $\left(8^{\prime \prime}\right.$ grarlo della salal Beauforll in lulla questa zonat e -ulle roste della peni-olal e dellat Si. 
ciliat. Alle 19th il fronte freddo -i inolta sul Timeno antro-meridionale; alle 0()$^{\prime}$ del 22 allatrersa in pieno, lungo un grande areo, la slessa zona e a!le $06^{\text {h }}$ procede verso es in direzione del continente. Le figure $5,6,7$ illustrano la siluzione sopra descrilla. La flg̣. 8 da jl corriepondente andamento dell'agitazione miero-i-mica. Anche in que-la figrua sono riporlate, a intervalli di 6 ore, 5 minuli di ree

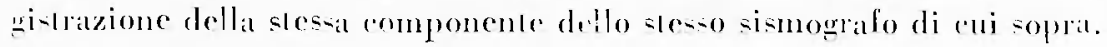

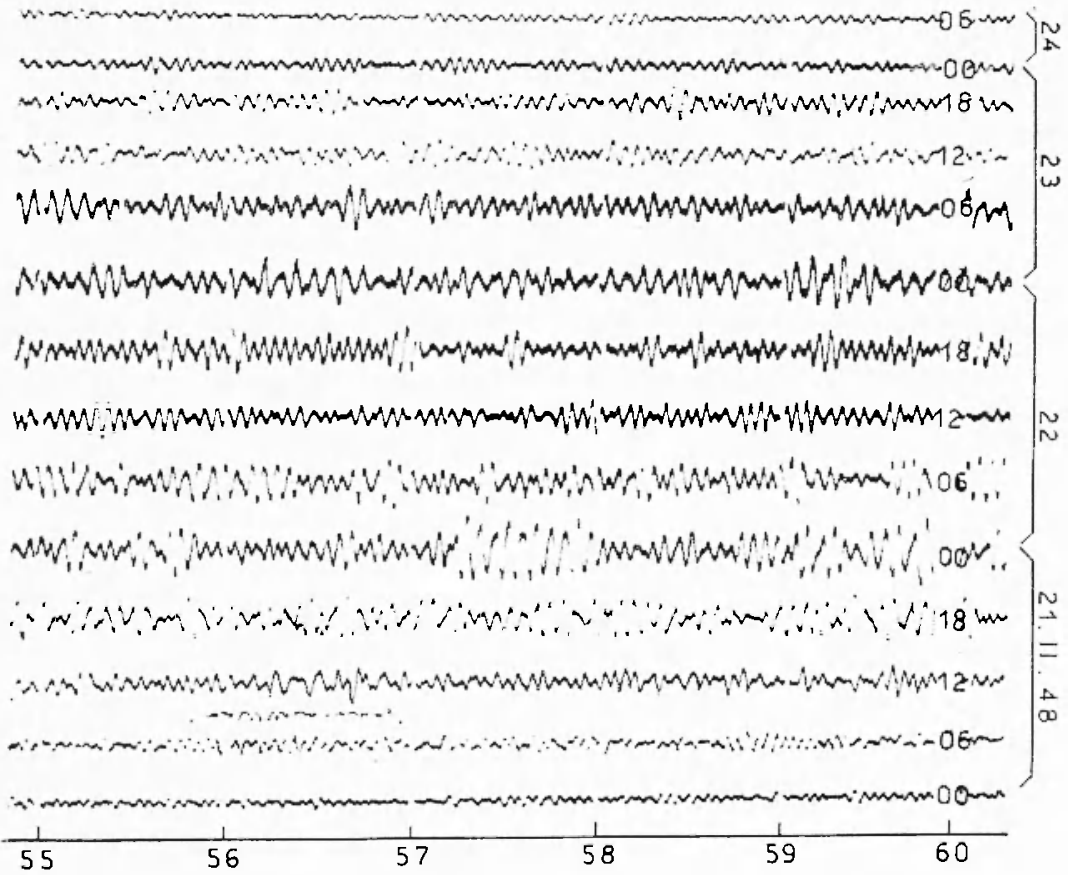

liig. 8

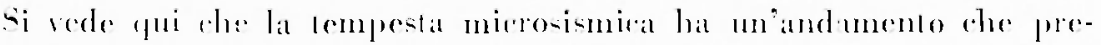

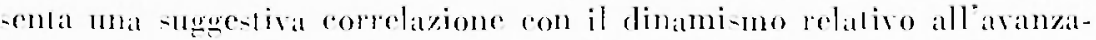
mento di un frome freddo -ulla regione di magriore profondita del Tirreno, ron un masimo di alliviti mierosismica coincidente con il par-

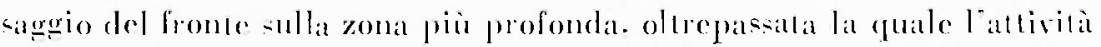
romat a diminuire sensibilmente e ancor più gquando oramai il fronie i passalo sul ronlinenle. Si può nolate un aumento del periodo ron

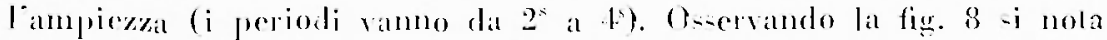




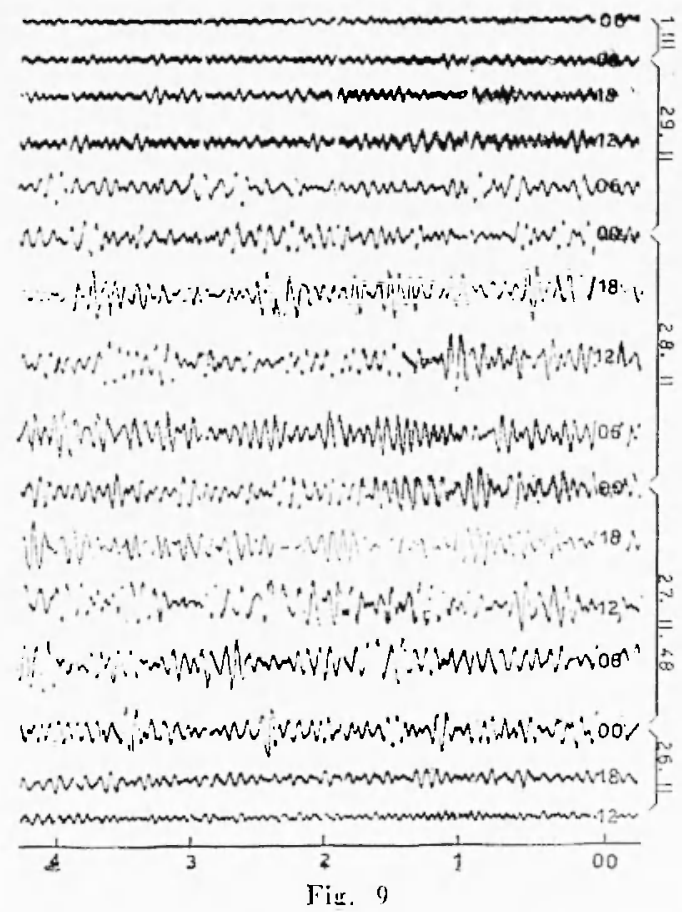

lalle I8 alle 2.0 ?h mal ma ripresil dellattivi. lit microsi-mitril en un periodo di $3^{\text {* }}$ ("il. elee lat un carallere un po" diverso dilal lia diversa orienes Esina $\therefore$ inlalli in corrispondenda di forli pereme baxioni nel golfo di Eenovar dove dalle $19^{h}$ del al a lat un malle tempentoso. Analoglor rondizioni si hamno nel mall Tiwhle insieme at forti rouli lin -ulla Gorriral. l'anilatione microsi-micil lral il 2.9 a il 3.3. di periodo piic lumgo mat non ben desermi-

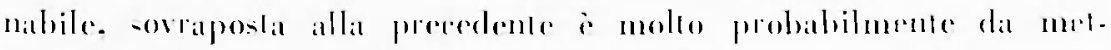

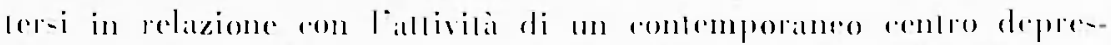
-ionario. ron un forte erartiente barieo. in prosimila delle Azzorre.

3) Tempesia microsismiea del $26-29$ febbraio 19.68. Nella lig.

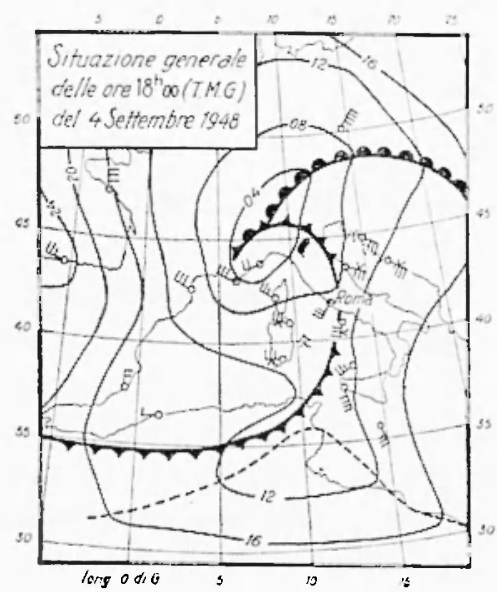

Fiig. I0 -ono riportale. a iulervalli di o ore. yualleo minuli di regi-lrazione lat

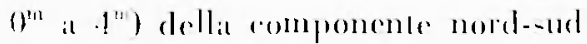

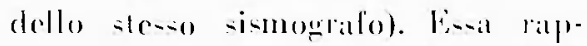
presenta un e-empice di allisili miero-i-miea di origine medilerraneat mat derivante dat comples. $=i$ fillori meleorologici inleremanti lullo il Medilerraneo entrale come dentale. romprero il mas Ionio. il liremo ed il mill ligure. Il giormo

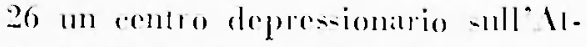
lantico entrale, rima-lo per yualehe lempo in rondixioni saxionater lat 
cominciato al avamalle in direzione est verso il rontinente entopeo od ha, surecesivamente, dalo luogo a due centri di eui uno ha seguito

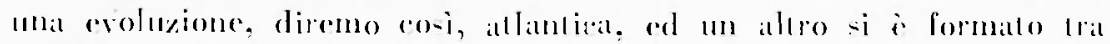
le Balcari, lat Spatgina e l'Africa seftentrionale. Dal que-to momento comincial a ri-contrarsi un forti-eimo gradiente barico, con venti ancheni forticimi, in lutlo il Mediterraneo compresi il Tirreno e lo Ionio. Il mare i grosso e tempe-toso nel Tirreno a nello Ionio, la

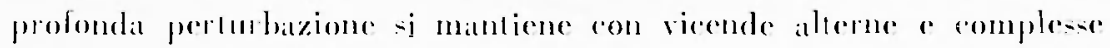

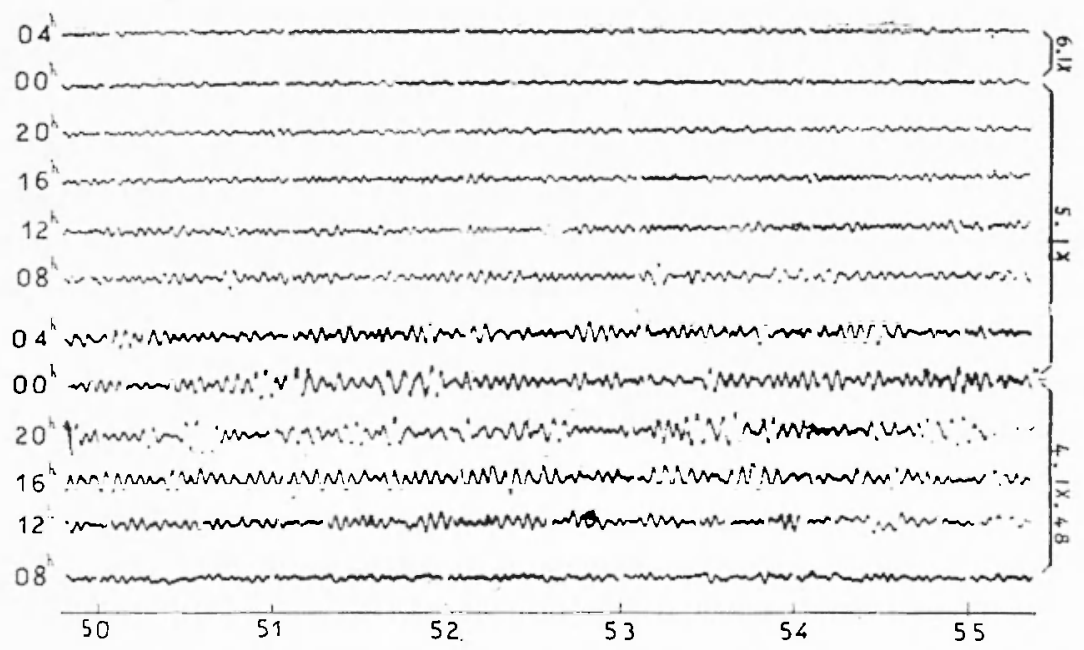

Fix. 1]

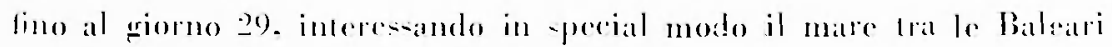
- lat Sardennat. il Tirreno a lo Ionio.

1) Tempesta mictosismica del 1.5 sellembre 19/3. Il griorno 1

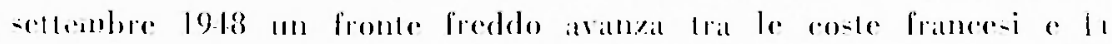
Corstrat a si reontrano renti molto Porli sperialmente sul Tirreno;

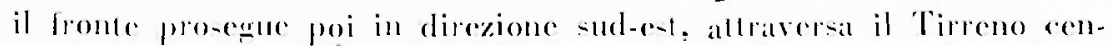
tre-meridionale (-i hamo ora renti forli-mimi, eperialmente in que-

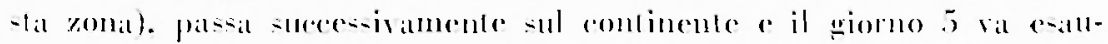
semolo la slla allivita. Lat fig. 10 da la -ituazione gencrale alle ore 18 del wiorno l.1 seltembre. I n confronte con lis ligurat rom-ente di met- 
tere in facile rilievo la sorplendente roneomitanza della massima ale livila mierosi-mica e del pasatgergo del fronte sulla zona più profonda del Tirteno.

Roma -- Istimuo Nazionale de Geofisica - Ouobre 1918.

\section{RI.LSSUTO}

Si fa un confronto doll agitaziome microsismica registrata in alcmni osservatori italiani della rete dell tstambo Vasionale di Coofision c si mellono i risulati in rappotto con la seologia locale: si passa allo sudio delle fondamentali caralteristiche dei mierosismi registrati a Roma in relazione con le permelazioni meteorologiche di origine

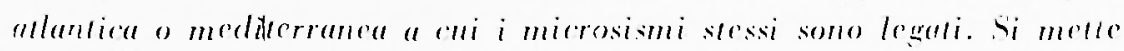
un ceidenza la importanza dello Ossertatorio di Roma nei rignardi specialmente della previsione de! empo nel bacino medierramen: st esaminano alewne tipiche tempeste mierosismiche registrale in epmesto os-

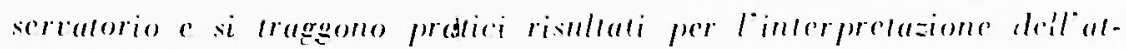
livilù mirrosismica.

In farticolare risulta che lagilazione caralleristica di 3 see cined. spesso di grande ampiezan, rhe iri si registra, is in correlazione an

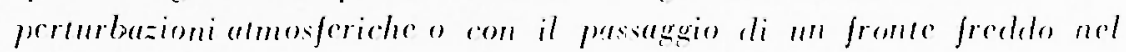
Timeno centro-meridionale rios nella zona piir profonda di questo mare.

\section{BIBLIOGRAHA}

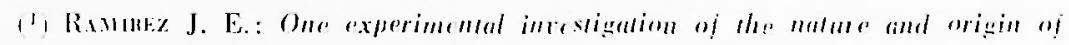
the microseisms at St. Louis - Bull. Sei-m. Sor. Am.. 30. 35-81 19901.

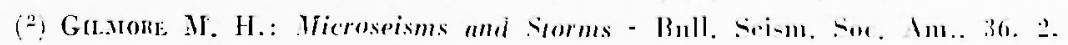
(9)-120 (19-16).

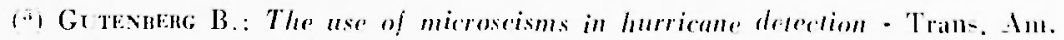
Geopluy Laion. 27 (19-46).

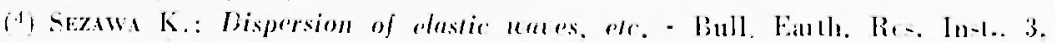
16 (1927).

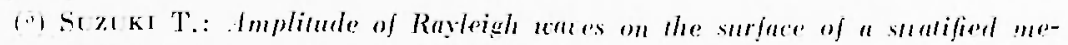
dirum - Bull. Earth. Res. In:l. II. 187-195 11933). 


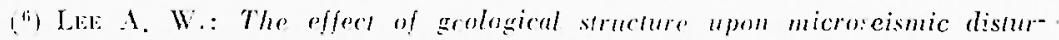
bunce - M.N.R.A.S. Geoplyys.. Suppl. 3 1]936.

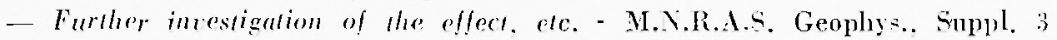
(1936).

(i) Grkxme; B.: Microseims and weather fordesting - The Jourual of Memonology. 1. $21-28$ (1917).

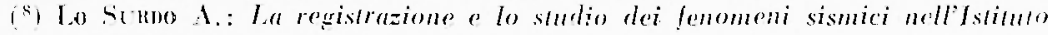
Sazionale ai Geofisica - La Ricerea Sidentifica n. 10 (19)f(1).

(") Gomor M. - Varde P. F.: Contributo allo smalio dille onde a M n - Imuli li Geofinira. I. 1 (19.18).

(10) Cinol P.: Comportameno delle onde di Rerleigh in un mezo firmo-ela-

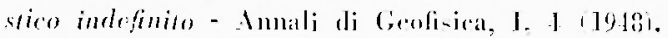

